\title{
ON PEER SELECTION AND PROTECTION FOR RELIABLE PEER-TO-PEER VIDEO STREAMING
}

\author{
Chi-Wen Lo ${ }^{*}$, Chia-Wen Lin ${ }^{*}$, Yung-Chang Chen ${ }^{*}$, and Jen-Yu Yu ${ }^{* *}$ \\ * Department of Electrical Engineering, National Tsing Hua University \\ 101, Section 2, Kuang-Fu Road, Hsinchu 30013 Taiwan \\ cwlin@ee.nthu.edu.tw \\ Information and Communications Research Laboratories \\ Industrial Technology research Institute \\ KevinYu@itri.org.tw
}

\begin{abstract}
P2P live video streaming is an emerging technique for video-ondemand services. In a P2P network, the bandwidth, memory, and computation ability of peers are redistributed to provide large-scale services. The key concept of P2P streaming is that each peer should forward the received data to its neighboring peers. However, peers might unexpectedly join and leave the P2P network, namely, peer churns, which can seriously disturb the video bitstream sharing. When a parent peer leaves, the receiving peers must find a replacement peer in time; otherwise, the receiving peers cannot resume their video transport. In addition, peers that connect to those receiving peers will also be influenced. This effect is known as chain reaction. In this paper, we propose a new P2P streaming method involving peer selection, video coding, and packet protection. Simulation results show that our proposed method can achieve better video quality and more reliable $\mathrm{P} 2 \mathrm{P}$ video streaming compared to existing schemes.
\end{abstract}

Keywords: P2P video streaming, peer selection, salable video coding, multiple description coding.

\section{Introduction}

P2P live streaming is an emerging video-on-demand (VOD) service. The major advantage of P2P-based VOD system is its lower cost of infrastructure than the clientserver-based and IP-multicast-based structures. Besides, user and network scalability can be achieved by distributed resource allocation of upload bandwidth, memory, and computing power among peers. In P2P video streaming, peers may have heterogeneous network conditions, e.g., diverse download/upload bandwidths and network delays, and channel conditions. Scalable video coding (SVC) is designed to transmit video data over heterogeneous networks, making it suited to be used in P2P video streaming services. With SVC, peers can adapt video quality according to peers' download bandwidth. Multiple description coding (MDC) divides a video streams into many individual substreams. The more the number of descriptions 
received at a client, the better the received video quality. This property of MDC can cope with peer dynamics, that is, when a peer loses one or more substreams due to peer dynamics, the peer still can obtain a baseline video quality. Because SVC substreams are dependant, losing lower-layer packets will cause higher video data non-decodable. But one drawback of MDC is its low coding efficiency. Shen et al. [1] showed that, in P2P video streaming, layered coding outperforms MDC, but only on no peer dynamic condition. A hybrid video coding scheme that combines the advantages of both coding methods would be beneficial.

Incentive-based method has recently attracted much attention in P2P research community. In such kind of mechanisms, peers are encouraged to distribute data to other peers so as to get a high credit rank. Higher ranking peers will receive better services than lower ranking ones. The metrics for credit ranking were often the number of uploaded packets or the upload bandwidth [2]. However, since video packets have dependency and are of different importance, they should be prioritized. Peers sending more important video data or contributing more channel bandwidth should be considered more important and should obtain better network resources.

When a peer leaves from or fails in a P2P network, its child-peers have to seek a replacement parent-peer to resume video delivery. If a child-peer fails to find a replacement peer on time, the video bitstreams will not be available for playback and re-distributing to decedent peers. More seriously, some peers may become unstable because of this chain reaction [3]. In this paper, we propose a parent/child-peer selection scheme for reliable P2P video streaming. Our scheme is kind of incentivebased algorithms in which ranking metric are defined to take into account the importance of video packets, the credits of video peers, and the reconstructed video quality. In our method, peers select their parent-peers and child-peers according to the proposed ranking metrics. The proposed method gives higher priority to peers that deliver more important video data (e.g., base layer), therefore they can resume video transport rapidly after suffering parent-peer churns. In addition, we also propose an adaptive protection method, by which high ranking peers will be allocated with more protection capacity to overcome data loss due to peer churns.

The rest of this paper is organized as follows. The proposed peer selection scheme and adaptive protection method are presented in Section 2. Section 3 shows the simulation settings and the results. Finally, conclusions are drawn in Section 4.

\section{Incentive-based P2P video streaming}

\section{$2.1 \quad$ Video format}

MDC has been widely studied for P2P video streaming systems. With MDC, a video stream is divided into several individual substreams (descriptions) so that a receiver can receive a base-quality video when receiving only part of the substreams. It is thus suitable to mitigate the impact of packet loss due to a poor channel condition or peer churn. MD-FEC applies unequal error protection (UEP) by which bits in more important video layers are given more protection. However the coding efficiency of MDC is typically less than that of SVC. Besides, SVC is more flexible than MDC in terms of scalability and video adaptation. On the contrary, the descriptions of an 
MDC video do not have the coding/decoding dependency as that existing in the layers of an SVC video, making MDC easier to tackle with network problems and to make better use of multipath diversity of a P2P network.

In this paper, we present a video coding structure that takes into account both MDC and SVC by modifying MD-FEC [4]. MD-FEC uses an FEC coding method, such as Reed-Solomon (RS) code to encode $k$ symbols and $M-k$ parity symbols so that the decoder can decode the $k$ original symbols as long as it receives any $k$ out of $M$ coded symbols. This coding strategy is also called $\operatorname{RS}(M, k)$ code.

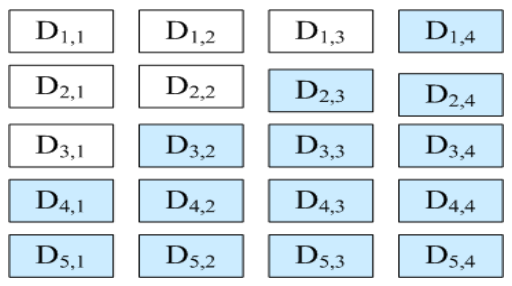

Fig. 1. The proposed MDC structure.

Fig. 1 depicts the proposed MDC structure where chunk $D_{i, j}$ denotes the $j$-th layer of the $i$-th description of a video. In this example, a video is first encoded into 4 layers by SVC, then the MD coder encodes the 4 video layers with unequal protection ability into 5 descriptions. The grey blocks indicate data blocks, and the white blocks indicate parity blocks. In MD-FEC, the unit of a peer can request is a description $i$ $\left(D_{i, j}, \quad j=1, . ., 5\right)$, whereas in our proposed method the requested unit can be down to a specific chunk $D_{i, j}$. The objectives of the proposed method are as follows:

1) High scalability: With MD-FEC, if a peer receives only partial descriptions, the higher layers in the received descriptions become useless because the higher layers are not decodable, thereby leading to bandwidth waste. In our proposed method, a peer can request the video layers according to the peer's available bandwidth, therefore bandwidth utilization can be made more efficient.

2) High bandwidth utility: For a P2P file sharing system, such as BitTorrent, a file is partitioned into several small segments, and a peer requests all these segments from different peers. This approach has shown promising performance in real $\mathrm{P} 2 \mathrm{P}$ file sharing applications. CoolStreaming [3], a real-world large-scale P2P streaming system, also adopts this concept. However, one description in MD-FEC is transmitted by one peer, and the bit-rate is about several hundred kbps. The uplink/downlink bandwidth resource is occupied by only few peers. Moreover, some low-bandwidth devices, i.e., mobile devices, cannot afford the enormous size of a description for downloading or uploading. In our proposed system the bitrate of $D_{i, j}$ is designed to be no more than $100 \mathrm{kbps}$. One description can be transmitted by many peers, and even the low-bandwidth device can contribute its bandwidth. Consequently, the bandwidth utility increases.

3) Flexible protection choice: Due to one chunk can be requested individually, the number of chunks in the same layer could be chosen strategically. For instance: the number of chunks in base layer can be much more than $k$, in this example $k$ is 2 , to against peer dynamics. 
Since an RS encoding/decoding procedure is not complicated when $M$ and $k$ are not large, a peer can re-encode all chunks in the same layer with $\operatorname{RS}(M, k)$ after receiving and decoding the partial chunks in the same layer-. For an example: when a peer received any two of $D_{i, 1}, i=1, . ., 5$, it can re-generate all of $D_{i, 1}$. This design can eliminate "collision" [4] which means that not all of candidate peers could be used, since there are identical chunks among peers. This leads to that peers cannot request wanted chunks from the candidate peers. With the proposed re-encoding scheme, a peer can request any chunk in the same layer from a peer.

\subsection{Proposed P2P Streaming Scheme}

Our P2P video streaming system adopts a mesh-based, push-pull P2P streaming structure. When a peer requests a P2P session, it first contacts the bootstrap server to obtain a peer list. The newcomer peer then "gossips" to the peers in the list to acquire information about other existing peers and video buffer map. As a result, the peer requests video chunks according to the buffer map. Besides, it can disconnect a streaming session by sending a "Leave" message to other peers. On the other hand, if a peer does not respond to gossip messages or requests of video chunks, the peer can be expected to be a failure peer, and those peers that connect to the failure peer have to find another replacement peer to resume the session. In the case that a peer cannot find a replacement peer in time during the leaving/failure of a parent peer, the peer's streaming session will be interrupted, which is called direct effect. Moreover, its descendant peers will turn to starvation, namely chain reaction. As depicted in Fig. 2, in the chain reaction, if peer $A$ leaves (the solid X), both peers $B$ and $C$ cannot receive video. In the direct effect, if peer $B$ leaves (the dotted $\mathrm{X}$ ), peer $C$ cannot receive the video, which can be resolved by using MDC. In this paper, we propose a peer selection scheme to protect important data as well as to reduce coding redundancy.

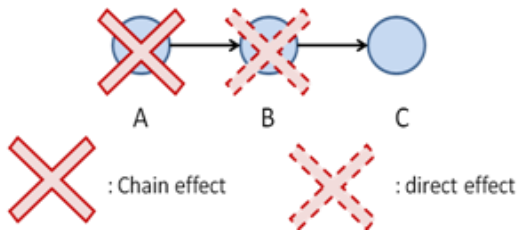

Fig. 2. Different effects caused by peer leaving.

The main concept behind the proposed peer selection scheme has two aspects:

1) Children selection: In incentive-based P2P streaming, a parent-peer should maintain good video quality for well-behaved children peers under the parent-peer's upload bandwidth constraint. A child-peer is considered well-behaved if it has been contributing more important video data or has delivered data to more peers. Generally, incentive-based methods encourage peers to share data with other peers; otherwise, poor-behaved child-peers will be punished by lowering down their utility. However, most the metrics used in existing incentive-based method for ranking a peer's contribution are the peer's upload bandwidth, and the amount of packet/video blocks the peer has contributed. Using such simple metrics may not be able to recognize a 
well-behaved peer in a video delivery path to serve them with better resource that it deserves. Suppose that peer $A$ has contributed more data than peer $B$, whereas $B$ has contributed more base-layer chunks than $A$. When a parent peer of $A$ and $B$ leaves, peers $A$ and $B$ have to find a replacement peer. In traditional incentive-based methods, $A$ will typically be offered with higher priority to resume its video data delivery than $B$. However, there may have more descendant peers of $B$ which may turn to starvation. To address this problem, we propose a new metric for ranking a peer's contribution that takes into account the video data dependency. We use the mean squared error (MSE) distortion of a video packet, which reflects the quality of reconstructed video, as a significant index. The distortion contributed by a video packet can be calculated off-line at the encoding stage, and it can be recorded in the packet header. Accordingly, a parent-peer evaluates the rank of a child-peer by

$$
S_{\text {child }}=\alpha \frac{\sum_{n} D_{n}^{\text {con }}}{D_{\text {parent }}^{\text {con }}}+(1-\alpha) \frac{\sum_{n} R\left(D_{n}^{\text {con }}\right)}{R\left(D_{\text {parent }}^{\text {con }}\right)}
$$

where $S_{\text {child }}$ is the score of the child peer, $D_{n}^{\text {con }}$ represents the distortion of the contributed video chunks from the child-peer, $R\left(D_{n}^{c o n}\right)$ denotes the rates of the contributed video chunks $D_{n}^{\text {con }}, D_{\text {parent }}^{\text {con }}$ and $R\left(D_{\text {parent }}^{\text {con }}\right)$ respectively represent the distortion and bandwidth contribution of the parent peer, which are used as normalization factors in (1). $\alpha$ is a factor ranging in $0 \sim 1$ to weight the contributions from the distortion and rate. In this work, we simply set $\alpha=0.5$.
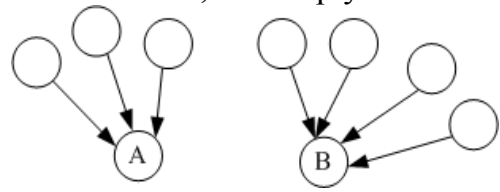

Fig. 3. Different ability parent peers.

In our method, each parent-peer keeps maximizing the sum of $S_{\text {child }}$ in a time period by kicking out the lowest ranking child-peers, and retaining/recruiting the high ranking ones. However, this can potentially cause the stream interrupt problem especially for newcomer peers, since they have no credit as they have not contributed any chunk yet. A start-up period $T_{s}$ is introduced such that a newcomer peer will not be kicked out within the start-up period, thereby allowing the peer to have time to accumulate credit so as to compete fairly with other existing peers.

2) Parent selection: Each child-peer should attach to parent-peers which can send video data to their descendants reliably. In current mesh-based P2P streaming methods, when a child-peer has several candidate parent-peers, it tends to connect to those with highest available upload bandwidth, or just randomly selects parent-peers. Such methods, however, cannot bear peer churns and can cause chain reactions easily. We take availability, the number of source chunks in a layer into consideration. Fig. 30 shows a parent-peer selection example. Assume that $\mathrm{RS}(5,3)$ coding is applied to a video layer such that once a peer receives at least 3 chunks, the other chunks can be regenerated correctly. For candidate parent $A$, if any one of its parent-peer fails, peer 
$A$ cannot RS re-encode chunks any more, leading to loss of video chunks. In this case, if one peer selects peer $A$ as its parent-peer, it will be very probably to starve. Under the same condition, peer $B$ is chosen to be a parent peer. Even if one of these parent peers fails, peer $B$ still can perform RS re-encoding. Therefore peer $B$ is a better choice, because it is resilient to the effect of grand-parent peers leaving so as to mitigate chain reaction.

In our method, peers with higher $S_{\text {child }}$ ranking will receive better services from its parent peers. As a result, their child-peer will also inherit better services from the higher ranking parent peers. Therefore, the rank of a candidate parent peer among grand-parent peers should also be taken into consideration. The proposed method jointly considers the availability, rank, and available download bandwidth as follows:

$$
\begin{gathered}
S_{\text {parent }}=A_{\text {parent }} \cdot\left(1-R_{\text {parent }}\right)+B W_{\text {available }} \\
R_{\text {parent }}=\frac{1}{M} \sum_{m} \frac{r a n k_{m}}{T_{m}}
\end{gathered}
$$

where $A_{\text {parent }}$ denotes the availability of the candidate parent-peer, $R_{\text {parent }}$ represents the average rank among grand-parents, $M$ is the number of grand-parent peers, $\operatorname{rank}_{m}$ is the parent rank in the $m$-th grand-parent, $T_{m}$ is the number of children peers of the $m$-th grand-parent peer, $B W_{\text {available }}$ is the available download bandwidth, and $R_{\text {parent }}$, rank $_{m}$ are from 0 to 1 . The parameter $B W_{\text {available }}$ allows a newcomer peer to acquire video chunks during the initial stage when the peer just joins a network and has not yet established it credit. Higher $S_{\text {parent }}$ rank implies higher stability of a peer. The ranking information can be attached into gossip messages. Chunks are requested from the lowest layer to high layers, therefore base layer chunks should be transmitted by the most reliable parent peers. Besides, high-layer chunks should not be requested if the available download bandwidth of the child peer is not affordable. Each child should keep maximizing the sum of $S_{\text {parent }}$ dynamically.

\section{Analysis of the Proposed Scheme}

The proposed parent/child-peer selection scheme assigns well-behaved peers with more reliable parent-peers. But these peers also suffer from direct effect. Automatic Repeat request (ARQ) based methods can resume the interrupted bitstream at the cost of introducing extra delay. On the other hand, MDC based methods do not induce delay but introducing extra redundancy. In our proposed MD-FEC scheme, redundancy data are generated according to a peer's importance. Important topological peers that carry more significant video chunks and contribute data to more peers will be protected more heavily. In what follows, we shall show that the proposed scheme is helpful in resolving chain reaction and in stabilizing the system. by

The probability of a video being un-decodable after peer churns can be modeled 


$$
p_{i}=\sum_{n=0}^{k-1}\left(\begin{array}{c}
k+d \\
n
\end{array}\right) \varepsilon^{k+d-n}(1-\varepsilon)^{n}
$$

where $k$ denotes the number of received chunks in the video layer that could be decoded by $\operatorname{RS}(M, k)$ coding, $d$ is the number of extra received chunks in the layer, and $\varepsilon$ is the probability of a parent peer leaving because of peer churn. In (4), peers receive $k+d$ chunks at most; however they may only receive less than $k-1$ chunks after peer churn. Therefore this video layer cannot be decoded anymore.

There are 3 possible situations when a peer becomes unstable. In situation A, namely adaption absorption, the unstable peer will find a new parent-peer with enough bandwidth to resume the chunk transmission. In situation B, namely adaption relay, the unstable peer finds a new parent-peer which, however, does not have enough bandwidth. The unstable peer wins the competition with new parent-peer's other children according to the score calculated by (1). A peer that fails in competing for a parent-peer has to find another new parent peer. In situation C, namely adaption amplification, the unstable peer cannot find any parent-peer with sufficient bandwidth or continuously loses peer competition. Not only the unstable peer remains unstable but also the $N_{c}$ children peers, which inherit the lost chunks, will also become unstable. Hence the number of unstable peers is increased to $N_{c}+1$.

The parent peer adaption can be modeled by a branching process. We focus on what parameters are important for overlay convergence. The reproduction rate $\xi_{q}$ of an unstable peer caused by peer churns can be formulated by

$$
\begin{aligned}
\xi_{q} & =p_{i}\left[0 \cdot P(A)+P(B)+\left(N_{c}+1\right) \cdot P(C)\right] \\
& =p_{i}\left[1-\mathrm{P}(\mathrm{A})+N_{c} \cdot P(C)\right]
\end{aligned}
$$

Its expected value becomes

$$
E\left(\xi_{q}\right)=p_{i}\left[1-P(A)+E\left(N_{c}\right) \cdot P(C)\right]
$$

where $P(A), P(B)$, and $P(C)$ denote the occurrence probabilities of situation $\mathrm{A}, \mathrm{B}$, and $\mathrm{C}$, respectively, as given by

$$
\left\{\begin{array}{c}
P(A)=1-p_{n s} \\
P(B)=p_{n s} \cdot\left(1-p_{R}\right) \\
P(C)=p_{n s} \cdot p_{R}
\end{array}\right.
$$

where $p_{n s}$ denotes the probability of a peer with insufficient upload bandwidth, and $p_{R}$, which can be calculated by (3), represents the probability of an unstable peer winning the competition with other children in the new parent. With the branch process, if $E\left(\xi_{q}\right)$ is less than 1 , the population of unstable peers finally becomes extinct after several generation steps. As a result, (6) becomes 


$$
\begin{aligned}
& E\left(\xi_{q}\right)=p_{i}\left[1-P(A)+E\left(N_{c}\right) \cdot P(C)\right]<1 \\
& \frac{1}{p_{i}}-p_{n s}>E\left(N_{c}\right) \cdot p_{n s} \cdot p_{R} .
\end{aligned}
$$

Equation (6) and (8) indicate it has high possibility to topology disruption if a high-contribution peer with large $N_{c}$ becomes unstable. However, our method leads to

$$
\begin{gathered}
S_{\text {child }} \propto N_{c}, \\
\left(1-p_{R}\right) \propto S_{\text {child }}, \\
d \propto N_{c} .
\end{gathered}
$$

In (8), peers with higher $N_{c}$ implies higher $S_{\text {child }}$, especially for base-layer chunks. Consequently these peers get higher distortion score. In (10) and (11), the higher contribution peer could less lose peer competition and get more protection chunks. This designs hold the inequality in (7), and reduce the unstable peers induced by the high contribution peers which state is unstable. The peer selection method by using (1), (2), and (11) not only emphasizes the reliability of a peer in the overlay topology, but also jointly considers the layer dependency and peer topological importance to diminish the chain reaction caused by peer churns.

\section{$4 \quad$ Simulation Results}

We conducted simulations including 2000 heterogeneous peers with five different downlink/uplink capacities as given in Table 1 [6]. Initially there are 1000 peers in the P2P network. These peers are self-organized into a mesh structure, and transmit video chunks based on the mesh topology. Peers then join in and leave from the network randomly at a join and leave rates of 120 peers $/ \mathrm{min}$, therefore there would be 1000 live peers in each time frame. We encode a CIF $(352 \times 288)$ video sequence at 30 frames/s by the scalable extension of H.264 (a.k.a. H.264 SVC) [7]. The scalable bitstream is coded into 10 descriptions, each containing 6 SNR layers. The bitrates and quality of each layer is listed in Table 2, where layer 0 means the base layer, and the others are the enhancement layers. These video layers are separated into many chunks to transmit, where the bitrate of each chunk is $50 \mathrm{kbps}$.

Table 1. Heterogeneous peers distribution

\begin{tabular}{|c|c|c|c|}
\hline Class & Downlink & Uplink & Percentage \\
\hline 1 & $512 \mathrm{kbps}$ & $256 \mathrm{kbps}$ & $56 \%$ \\
\hline 2 & $1.5 \mathrm{Mbps}$ & $384 \mathrm{kbps}$ & $21 \%$ \\
\hline 3 & $3 \mathrm{Mbps}$ & $896 \mathrm{kbps}$ & $9 \%$ \\
\hline 4 & $20 \mathrm{Mbps}$ & $2 \mathrm{mbps}$ & $3 \%$ \\
\hline 5 & $20 \mathrm{Mbps}$ & $5 \mathrm{mbps}$ & $11 \%$ \\
\hline
\end{tabular}


The metrics we use for evaluating video quality include playback continuity, average decodable PSNR, and the variation of decodable PSNR. The continuity metric is defined as follows:

$$
\text { Cont }=\frac{\text { Number of decodable GOPs }}{\text { Total number of GOPs }}
$$

For an SVC coded bitstream, if the base-layer of a GOP is decodable, the GOP can be played back with baseline quality. Therefore the continuity metric is a measurement of the reliability of the base-layer data being received. The average decodable PSNR is defined as

$$
\operatorname{avgPSNR}=\frac{1}{L} \sum_{n=1}^{L} \operatorname{PSNR}(m, n)
$$

where $L$ is the on-line time of this peer. Since peers may not successfully receive enhancement layer chunks, the decodable video quality would fluctuate with the number of lost video chunks. Hence we evaluate video quality variation by

$$
s t d_{-} P S N R=\operatorname{STD}(P S N R(m, n)), n \in(1, L)
$$

Table 2. Bandwidths and average PSNR qualitites of heterogeneous peers

\begin{tabular}{|c|c|c|c|c|c|}
\hline Layer & $\begin{array}{c}\text { Rates } \\
(\mathrm{kbps})\end{array}$ & PSNR & Layer & $\begin{array}{c}\text { Rates } \\
(\mathrm{kbps})\end{array}$ & PSNR \\
\hline 0 & 150 & $28.93 \mathrm{~dB}$ & 3 & 300 & $36.99 \mathrm{~dB}$ \\
\hline 1 & 200 & $31.14 \mathrm{~dB}$ & 4 & 300 & $38.13 \mathrm{~dB}$ \\
\hline 2 & 250 & $33.99 \mathrm{~dB}$ & 5 & 300 & $40.35 \mathrm{~dB}$ \\
\hline
\end{tabular}

We compare three strategies: (a) a non-incentive-based method that implements random parent/child-peer selection without data protection, denoted as "random"; (b) an incentive method that choose child-peers according to the contributed upload bandwidth of peers, chooses parent-peers according to the maximum available upload bandwidth, and implements adaptive data protection, denotes as "maxBW"; (c) Our proposed incentive peer selection scheme and with adaptive data protection, denoted as "maxReliable."

Table 3. Heuristic algorithm to decide the amount of protection data.

$$
\begin{aligned}
& \text { if }(0<\text { distributed_count }[j] \& \& \text { distributed_count }[j] \leq 5) \\
& \quad \text { redundancy }[j]=1 ; \\
& \text { if }(5<\text { distributed_count }[j] \& \& \text { distributed_count }[j] \leq 10) \\
& \quad \text { redundancy }[j]=2 ; \\
& \text { if }(10<\text { distributed_count }[j] \& \& \text { distributed_count }[j] \leq 20) \\
& \quad \text { redundancy }[j]=3 ;
\end{aligned}
$$




$$
\begin{gathered}
\text { else if }(20<\text { distributed_count }[j]) \\
\text { redundancy }[j]=4 ;
\end{gathered}
$$

Since developing an optimized channel protection method is not the central focus of this work, we here use a heuristic algorithm to decide the amount of the redundancy data, as shown in Table 3, where distributed_count $[j]$ denotes the number of delivered churns for the $j$-th layer, and redundancy[j] is the number of additional churns for data protection (i.e., the parameter $d$ in (4)). The key idea behind the protection algorithm is that more protection data in layer $j$ should be given to the peers which contributed more churns in layer $j$ such that these peers can remain stable under peers churns. In addition, these peers serve as the reliable parent-peers to delivery video data. For fair comparison, the algorithm is applied in both " $\max B W$ " and "maxReliable."

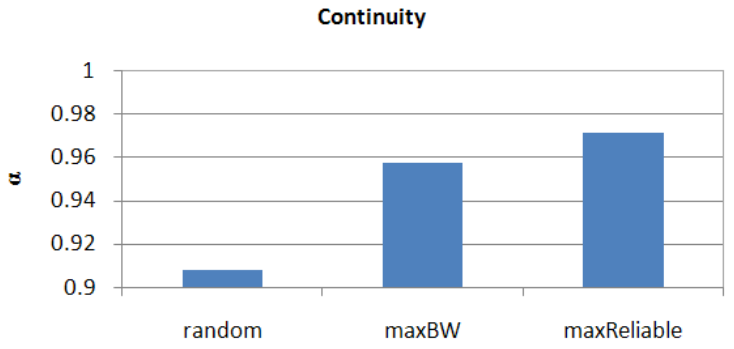

(a)

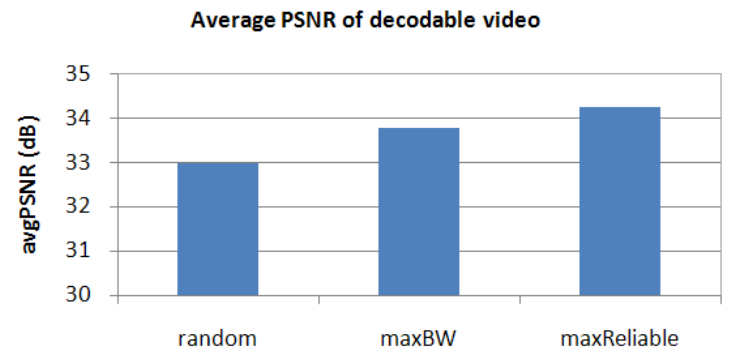

(b)

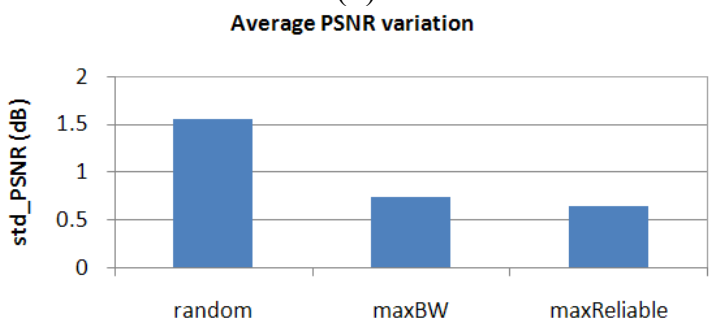

(c)

Fig. 4. $\mathrm{P} 2 \mathrm{P}$ video performance on (a) continuity, (b) PSNR of decodable video, and (c) PSNR variation. 
Fig. 4 compares the overall system performance. Random peer selection is a wellknown effective method implemented in commercial $p 2 p$ video streaming systems, such as CoolStream [3]. Compared to the random selection, our adaptive selection method achieves more reliable video quality in terms of continuity, especially for "maxReliable," as shown in Fig. 4(a). Because "maxReliable" ranks peers by the distortion of contributed video chunks, the parent-peers with high contributions in delivering base-layer chunks will be given more protection. As a result, their childpeers will have reliable base-layer sources, thereby mitigating the chain effect. On the other hand, " $\max B W$ " uses the amount of contributed bandwidth to rank a peer cannot fully recognize the peer's importance in a video delivery path, therefore the protection resources may be improperly allocated to less important peers.

Continuity

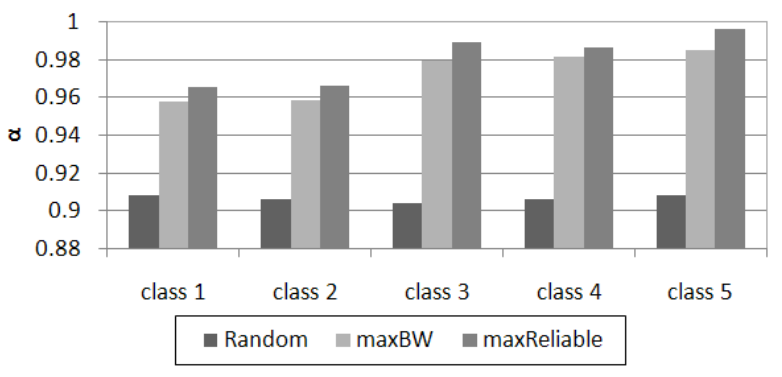

(a)

Average PSNR of decodable video

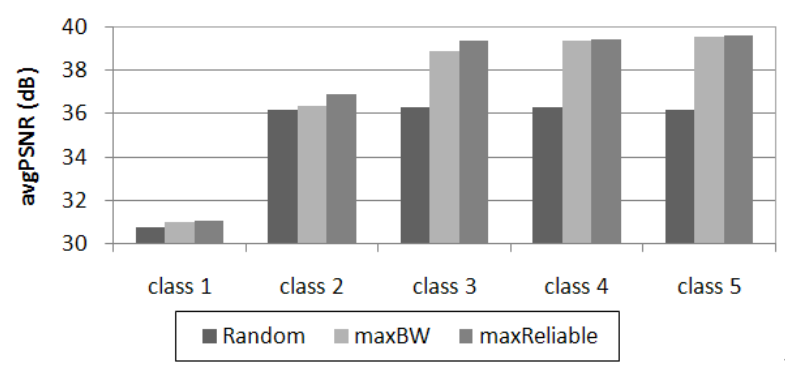

(b)

Average PSNR variation

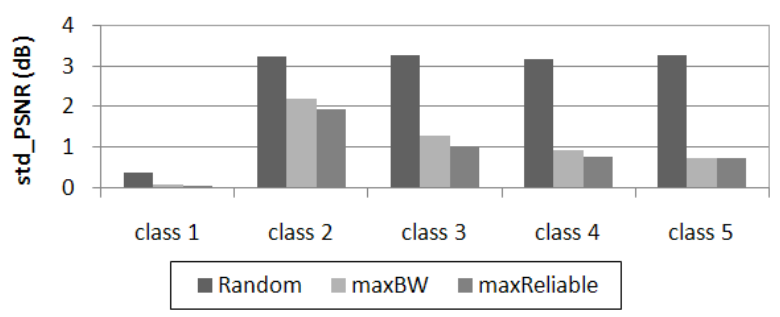

(c) 
Fig. 5. Performance comparison for different peer classes in terms of (a) continuity, (b) PSNR of decodable video, and (c) PSNR variation.

Fig. 4(b) and (c) compares the PSNR performances of three methods. A high PSNR of a decodable video implies that the succeeding enhancement layers are also correctly decoded. Should the middle enhancement layers get lost, the video quality will drop, and the succeeding enhancement layers become useless. Fig. 4(b) shows that "maxReliable" outperforms other methods in the PSNR of decodable video, because it use the unequal scores of the contributed chunks to provide proper peer selection and data protection to peers. When the enhancement layers are lost, peers will find replacement parent-peers to resume the transmission of lost chunks. Frequent losses of enhancement-layer data will lead to fluctuation of video quality and cause uncomfortable human perceptual experience. As shown in Fig. 4(c), the quality variation of "maxReliable" is $41.28 \%$ of "Random", and $87.5 \%$ of "maxBW". The results show that "maxReliable" provides most reliable and smooth video quality.

Fig. 5 compares the performances of three different methods for different peer service classes. "Random" does not offer differential services in all quality measurements. Due to their limited download bandwidths, the "class 1" peers receive lower quality video with lower PSNR variation compared to the other classes of peers. With the "maxReliable" method, peers in a higher class receive higher continuity, higher PSNR quality video with better continuity and lower PSNR variation. Moreover, these peers, when serving as parent-peers, can provide their child-peers with more reliable video quality.

\section{Conclusion}

In this paper, we proposed an incentive-based peer selection method for reliable P2P scalable video streaming. We have also proposed a modified MD-FEC scheme which takes into account both video scalability and efficient bandwidth utilization. In our proposed method, video layer dependency and peer topology information are jointly considered to combat against chain reactions caused by peer churns. We adopted video distortion as the metric of our incentive method for child-peer selection. In parent-peer selection, availability and rank are applied to find robust parent peers. The proposed adaptive protection scheme avoids the direct effect of peer leaving as well as mitigates the chain effect. Simulation results show the proposed method improves video playback continuity and achieves better PSNR quality when peer churns occur.

\section{References}

1. Y. Shen, Z.Liu, S. S. Panwaret, K. W. Ross, and Y. Wang: Peer-driven video streaming multiple descriptions versus layering. In: Proc. IEEE Int. Conf. Multimedia Expo. IEEE Press, New York (2005)

2. Z. Liu, Y. Shen, S. S. Panwar, K. W. Ross, and Y. Wang: P2P video live streaming with MDC providing incentives for redistribution. In: Proc. IEEE Int. Conf. Multimedia Expo. IEEE Press, New York (2007)

3. S. Xie, B. Li, G. Y. Keung, and X. Zhang: Coolstreaming design, theory, and practice. In: IEEE Trans. Multimedia, vol. 9, no. 8, pp.1661-1671. IEEE Press, New York (2007) 
4. R. Puri and K. Ramchandran: Multiple description source coding through forward error correction codes. In: 33rd Asilomar Conf. Signals, Syst. Computers (1999)

5. Z. Liu, Y. Shen, S. S. Panwar, K. W. Ross, and Y. Wang: Efficient substream encoding and transmission for P2P video on demand. In: IEEE Packet Video Workshop 2007, pp.143-152. IEEE Press, New York (2007)

6. E. Setton, P. Baccichet, and B. Girod: Peer-to-peer live multicast: A video perspective. In : Proc. IEEE, vol. 96, no. 1, pp. 25-38. IEEE Press, New York (2008)

7. ITU-T and ISO/IEC JTC1, JVT-O202, Joint Scalable Video Model JSVM-2 (2005) 